\title{
Press-fit acetabular cup fixation: principles and testing
}

\author{
W Macdonald ${ }^{1 *}$, L V Carlsson ${ }^{1}$, G J Charnley ${ }^{2}$ and C M Jacobsson ${ }^{1}$ \\ ${ }^{1}$ Department of Biomaterials/Handicap Research, Institute for Surgical Sciences, University of Göteborg, Sweden \\ ${ }^{2}$ Derriford Hospital, Plymouth, Devon, UK
}

\begin{abstract}
Pre-clinical testing of the fixation of press-fit acetabular components of total hip prostheses relies on cadaver or synthetic bone, but the properties and geometry of bone models differ from those of physiological bone. Cup designs use varied mechanisms for initial stability in bone; therefore, using different analogues and tests is appropriate.

Press-fit cup stability was tested in the following: firstly, polyurethane (PU) foam modelling cancellous support; secondly, glass-fibre reinforced epoxide (GFRE) tubes modelling acetabular cortical support; thirdly, cadaveric acetabula. Three commercial cups [Harris-Galante II (H-G II), Zimmer; Optifix, Smith \& Nephew, Richards; porous coated anatomic (PCA), Howmedica] and an experimental cup with enhanced rim fixation were tested in three modes: direct pull-out, lever-out and axial torque.

The fixation stabilities measured in the PU and the GFRE models showed trends consistent with those in cadaver bone, differing in the oversizing and cup geometry. The experimental cup was significantly more secure in most modes than other cups; the H-G II and Optifix cups showed similar stabilities, lower than that of the experimental cup but greater than that of the PCA cup (analysis of variance and Tukey's highly significant difference test; $p<0.001$ ). The stabilities measured in cadaver bone more closely approximated those in GFRE. The use of several bone analogues enables separation of fixation mechanisms, allowing more accurate prediction of in vivo performance.
\end{abstract}

Keywords: arthroplasty, acetabulum, fixation, testing, mechanics

\section{NOTATION}

\section{Abbreviations}

GFRE

$\mathrm{H}-\mathrm{G}$ II

PCA

PU

Tukey's HSD glass-fibre reinforced epoxide

Harris-Galante II ( $\left({ }^{\circledR}\right.$ Zimmer Inc.)

porous coated anatomic

( ${ }^{\circledR}$ Howmedica Inc.)

polyurethane

Tukey's highly significant difference

\section{INTRODUCTION}

Pre-clinical testing of acetabular components of total hip replacements is essential, but current methods remain unproven and unconvincing. Cadaveric pelves are the most realistic test bed [1-3], but supply can be problematic and often the age and quality of specimens available

The MS was received on 19 February 1998 and was accepted after revision for publication on 27 July 1998.

* Corresponding author: Department of Biomaterials/Handicap Research, Institute for Surgical Sciences, University of Göteborg, Medicinaregatan 8, S-41390 Gothenburg, Sweden.

H00998 (C) IMechE 1999

are unrepresentative when compared with normal joint replacement populations.

Bone analogues have been used as test beds $[\mathbf{4}, \mathbf{5}]$, but different material properties and distribution may alter the fixation effects compared with those in vivo. Rather than seeing this as a disadvantage, it is postulated that using two vastly different bone analogues for acetabular cup testing might clarify some fixation effects in pressfit acetabula. To test this, two different bone analogues were used for acetabular cup testing and compared with cadaver bone.

\subsection{Fixation mechanics}

The first question in press-fit cup testing is: what mechanisms of fixation need to be tested? Adler et al. [4] concluded that 'the most important factor in determining cup mechanical stability was the ability of the cups to engage around the outer periphery'. This 'ability to engage' is a combination of the rim or edge form and finish of the cup, and the elasticity of the bone around the rim. Other mechanisms which contribute to the press-fit cup stability are the friction between the bone 
and the cup surfaces, and geometric effects; a cone in a conical cavity is more stable to tilting than is a hemisphere in a hemispherical cavity.

Designs of acetabular components rely to varying extents on these separate mechanisms. The fixation achieved in vivo will also vary with acetabular anatomy and preparation, altering the combination of mechanisms operating. These separate mechanisms will be differently represented by different test modes and test materials.

\subsection{Test modes}

The second question in testing of press-fit cups is: what failure mode is being represented? Whether micromotion leading to fibrous fixation is under consideration, or gross loosening accompanied by gross movement of the implant is postulated, the problem is how such movements are generated and resisted. Failure in rotation will be resisted by axial torque resistance, which depends primarily on surface friction effects. Lever-out due to impingement or articulation forces is resisted by a combination of friction, rim effects and shape or form contributions. Although axial pull-out is unlikely, pistoning in the acetabulum may provoke loosening, and resistance to this depends primarily on rim effects. The stability of cups relying differently on these factors will vary in bone and bone analogue models, especially if the mechanics and morphology of the analogue differ from the physiological behaviour.

\subsection{Test material}

Polyurethane (PU) foam is well accepted in surgical training, its cutting and threading behaviour being a good analogy of bone. However, the stiffness, distri- bution, frictional and yield behaviour are markedly different from those of acetabular bone, especially if surgical reaming leaves the subchondral shell intact.

The acetabulum can be modelled more properly as a thin hemispherical shell of subchondral cortex supported by cancellous bone and a conical shell of cortical bone $[6,7]$ with the cortical shell playing the more important role in the mechanical behaviour. In contrast with PU foams, fibre-reinforced materials show a closer resemblance to cortical bone physical properties and can be engineered to give mechanical, frictional and distribution properties similar to those of the anatomical cortex. It is believed that using a tube of fibre-reinforced plastic to model the cortical support of the acetabulum would better model the mechanics of the acetabulum.

Further it is postulated that by testing in PU foam blocks and fibre-reinforced epoxide cavities, using a range of tests and a variety of cup designs, it would be possible to compare the fixation strength and so further understanding of the fixation mechanics of a range of press-fit acetabular cups.

\section{METHODS}

Three acetabular cups reflecting the variation in overall form and surface finish in current clinical usage were obtained from operating theatres: Harris-Galante II (H-G II) ( $50 \mathrm{~mm}$ diameter, Zimmer Incorporated, Warsaw, Indiana), Optifix (50 mm diameter, Smith \& Nephew Richards, Memphis, Tennessee) and porous coated anatomic (PCA) (49 mm diameter, Howmedica Incorporated, Rutherford, New Jersey) (Fig. 1). To contrast rim effects more strongly, an experimental cup was produced with a rim shape and finish, enhancing the rim fixation. This cup was also produced with a $50 \mathrm{~mm}$ diameter. For test coupling, a shaped metal block was fitted

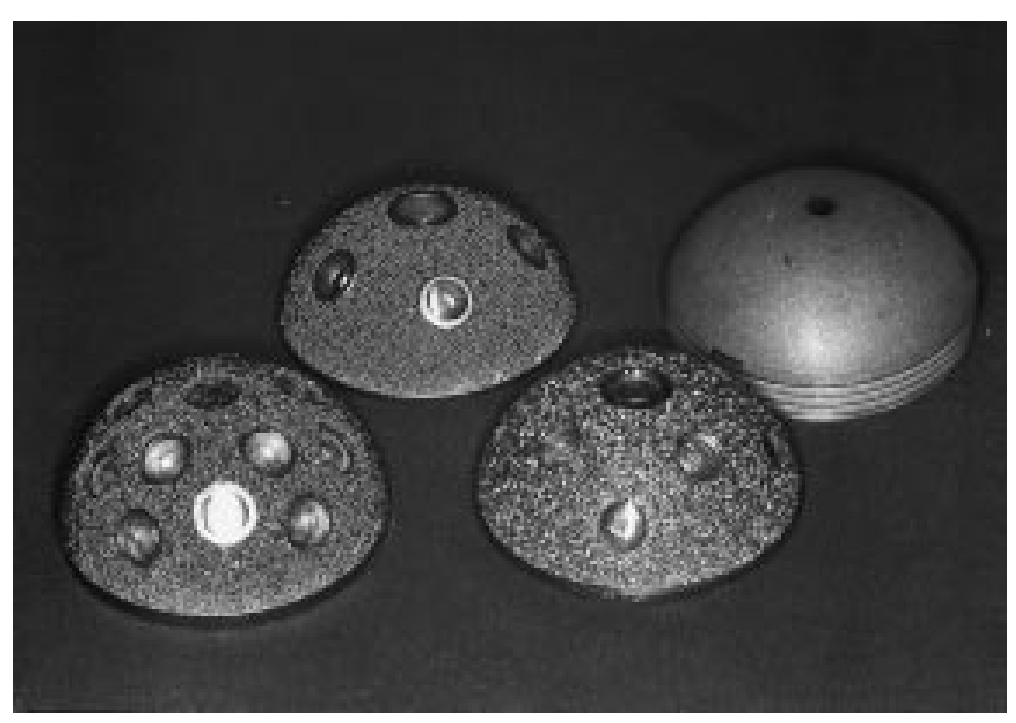

Fig. 1 Range of cups tested: from left to right, Optifix, PCA, H-G II and experimental 
into the bore of each cup and secured with epoxy adhesive (Araldite, Evode Limited, Stafford, UK).

The PCA cup has a full hemispherical form and sintered bead coating, with a soft rim. The Optifix also has a full hemispherical form and sintered bead coating but a more prominent rim. The $\mathrm{H}-\mathrm{G}$ II is compressed wire coated, less than the hemispherical form, and has a sharp rim. The experimental cup was designed with a full hemispherical form and a rim of cylindrical section with enhanced fixation. A solid surface grit-blasted finish was retained all over to enhance surface friction against bone.

Standard orthopaedic PU foam blocks (density, $0.2 \mathrm{~g} / \mathrm{cm}^{3}$, Sawbones Europe AB, Malmö, Sweden) were reamed with debris-retaining reamers of diameter $2 \mathrm{~mm}$ less than the cup diameter, in accordance with the supplier's instructions. Reamers and blocks were mounted in an engineering milling machine to ensure steadiness and accuracy in reaming, and cavities were measured with vernier calipers after reaming to check the diameter.

Glass-fibre reinforced epoxy (GFRE) with an elastic modulus of $20 \mathrm{GPa}$ (RLG/3, Tufnol Limited, Birmingham, UK) was produced in the form of tubes which were machined to a standard wall thickness of $1.50 \mathrm{~mm}$ and inside diameter appropriate for each cup. The tubes were clamped on a closely fitting support base with a concave polyethylene insert to complete the acetabular shape (Fig. 2). For lever-out and pull-out the tubes replicated the grip at the rim found in the physiological acetabulum, but the actual bone-metal contact (being cylindrical) was much less than the hemispherical surface contact in bone and PU foams, and thus torque testing (which is much more dependent on surface area) was not considered worthwhile.

Twenty-four cadaveric acetabula were also prepared for experimental cup insertion in mortuo. The standard

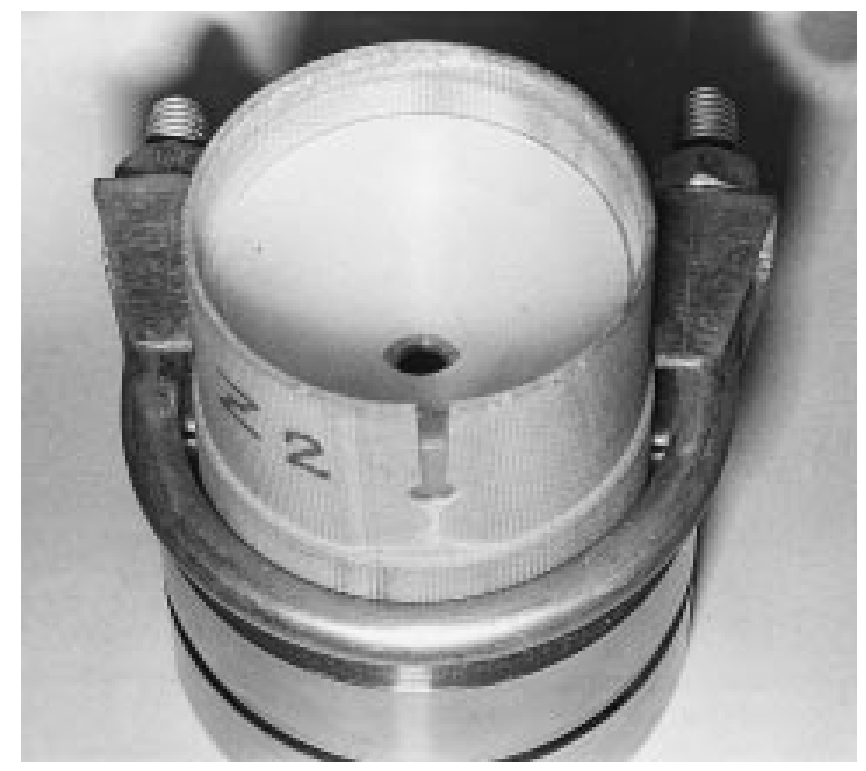

Fig. 2 GFRE tube mounted on a holder and cavity base support hip arthroplasty procedure was followed, exposing the acetabulum and reaming with progressive sizes of reamer, commencing with a small-diameter reamer cutting down to the true acetabular floor and then continuing to increase reamer diameter until the subchondral plate was being exposed and cut. Then a cup $2 \mathrm{~mm}$ oversized was inserted into the cavity.

Cups were impacted into position within the test cavity and tested in torque resistance, lever-out strength, or axial pull-out using purpose-designed rigs and a universal materials-testing machine (model 4301, Instron Limited, High Wycombe, UK). Pull-out tests were performed at a cross-head speed of $1 \mathrm{~mm} / \mathrm{min}$, lever-out tests with a lever-link of $250 \mathrm{~mm}$ from the cup centre and a cross-head speed of $60 \mathrm{~mm} / \mathrm{min}$ (Fig. 3), and torque tests at a rotational speed of $0.038 \mathrm{rad} / \mathrm{min}$. After each test the cup was cleaned, was inspected to ensure that no damage had occurred and was inserted into a fresh cavity, testing five fresh cavities for each cup in each mode. The mean strengths (and standard deviation) for each combination were presented in Figs 4, 5 and 6.

Cadaver tests were performed with portable test rigs reproducing the same conditions. Torque tests were

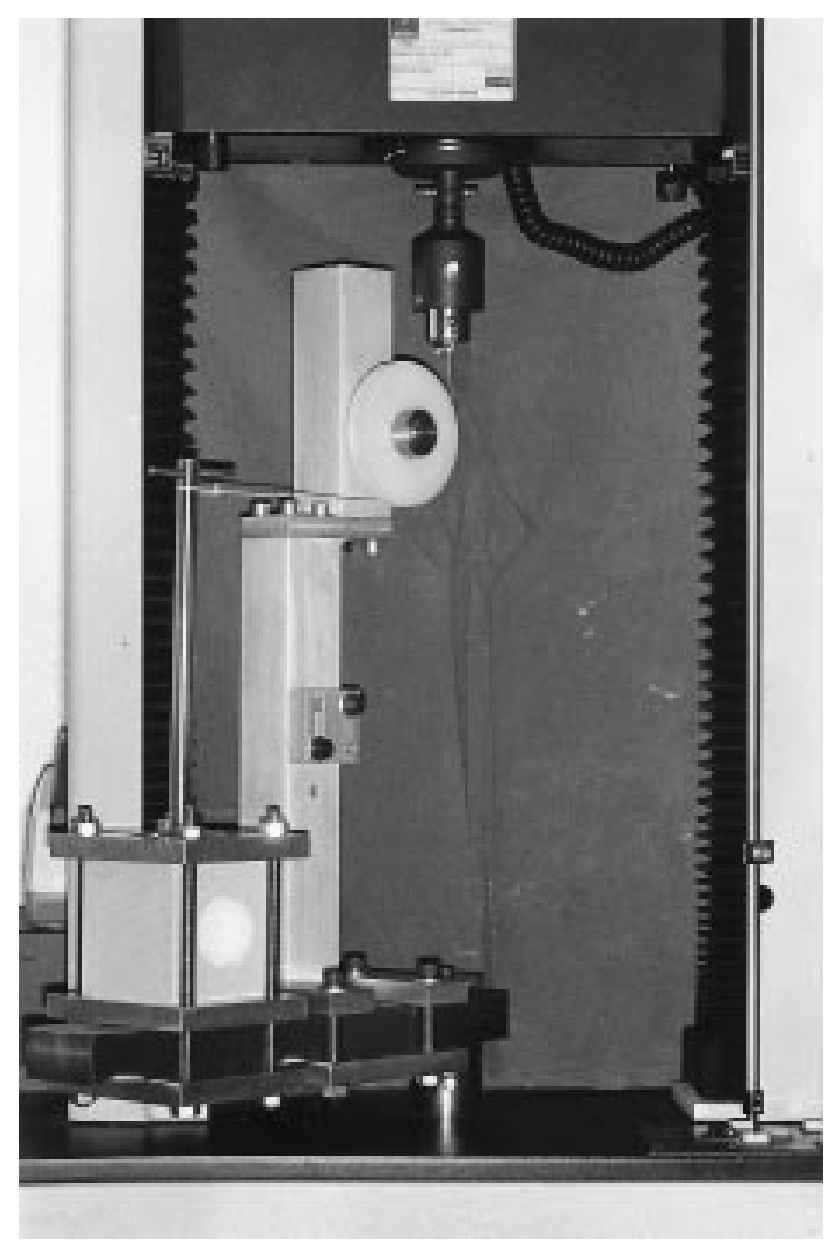

Fig. 3 Lever-out testing in the Instron materials-testing machine 


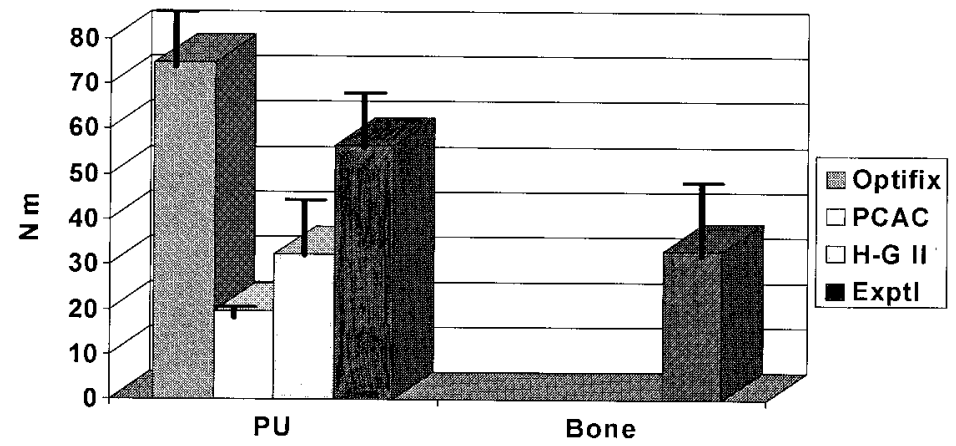

Fig. 4 Torque strengths by cup and material (mean + one standard deviation)

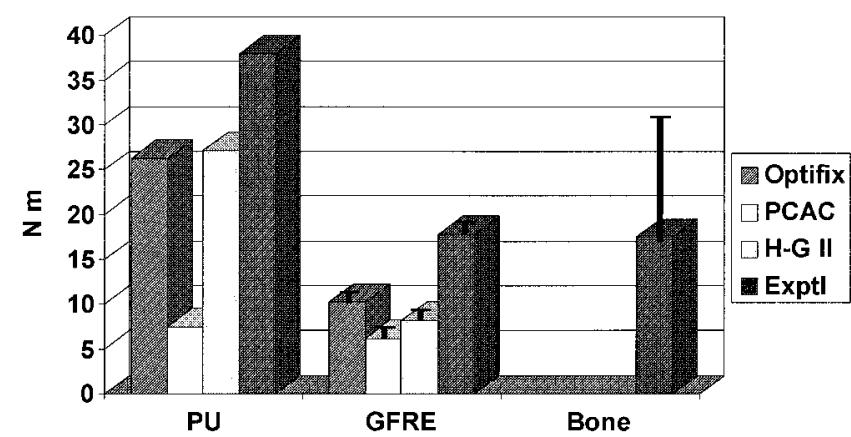

Fig. 5 Lever-out strengths by material and cup (mean + one standard deviation) (standard deviations in PU are so small that error bars are not shown)

undertaken with a torque transducer $(10 \mathrm{kgf} \mathrm{m}$ model LT-10KA, TML, Tokyo Sokki Kenkyujo Company Limited, Tokyo, Japan) coupled to the cup by a universal joint which did not permit the transmission of axial forces and allowed only pure torque, the torque being applied by hand. Lever-out was tested by screwing a $250 \mathrm{~mm}$ lever arm into the bore of the cup and applying a perpendicular force at the end of the arm with a maximum-recording spring balance. Pull-out tests used a ball-screw jack mechanism pulling against a tripod mounted onto the pelvis. The action of the jack and its linkages were aligned perpendicular to the central axis of the acetabular cup, the force developed being measured by the torque transducer indicating the torque required and it was calibrated against an electromechani- cal force transducer (500 kgf model U4000, Maywood Instruments Limited, Baskingstoke, UK). Data were recorded real-time using an analogue-digital converter linked to a lap-top computer.

GFRE cavities fractured on insertion of cups oversized to greater than $1 \mathrm{~mm}$, and therefore two vertical cuts were introduced into each tube, diametrically opposite and extending half the depth of the tube. Diameters were standardized to accept $1 \mathrm{~mm}$ 'oversizing' for all cups. To account for the oversizing differences, the experimental cup was further tested in PU foam blocks (Sawbones) at 1, 1.5 and $2.0 \mathrm{~mm}$ oversizing (Table 1) to compare the effect on the fixation strength. Oversizing was investigated over this range only, as misfit and poor contact due to greater oversizing are well proven $[4,8]$.

Statistical analysis was undertaken with a three-way analysis of variance and Tukey's highly significant difference (HSD) post hoc comparisons using the Statistica package (StatSoft, Inc., Tulsa, Oklahoma).

Table 1 Cup fixation strengths in PU foams with various oversizing (using the experimental cup)

\begin{tabular}{cccl}
\hline $\begin{array}{c}\text { Oversizing } \\
(\mathrm{mm})\end{array}$ & $\begin{array}{c}\text { Pull-out } \\
(\mathrm{kN})\end{array}$ & $\begin{array}{c}\text { Lever-out } \\
(\mathrm{N} \mathrm{m})\end{array}$ & $\begin{array}{c}\text { Torque } \\
(\mathrm{N} \mathrm{m})\end{array}$ \\
\hline 2.0 & 2.009 & 37.94 & 56.2 \\
1.5 & 1.482 & 33.4 & 31.6 \\
1.0 & 1.313 & 23.15 & Not tested \\
\hline
\end{tabular}

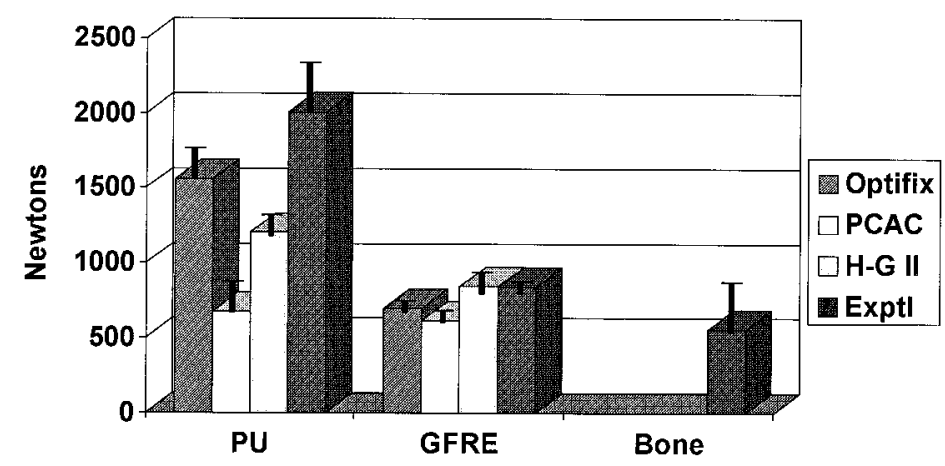

Fig. 6 Pull-out strengths by material and cup (mean + one standard deviation) 
Multiple-regression analysis was also used to test for systematic trends of cups or materials.

\section{RESULTS}

Fixation stabilities measured in PU foams, GFRE bone analogues and cadaveric bone showed comparable strengths, considering all factors influencing the measures. In PU foams, the Optifix cup was strongest in torque, the experimental cup was strongest in pull-out and lever-out, and the PCA showed lowest strength in all tests (Figs 4 to 6 ). The pull-out strength was greatest in the experimental cup and then in the order Optifix, H-G II and PCA but, in lever-out, Optifix and H-G II were equal after the experimental cup. In GFRE, the H-G II and experimental cups were slightly stronger than the others in pull-out but, in lever-out the H-G II was third after the experimental (the strongest) and Optifix cups. Cadaver trials gave a wider variation in results than laboratory tests did (Figs 4 to 6), and lower strengths. Lever-out and pull-out of cups with strong rim fixation (the $\mathrm{H}-\mathrm{G}$ II, Optifix and experimental cups) often removed a conical annulus of PU foam during testing (Fig. 7). The size of annulus removed varied with cup design; the stronger rim fixation of the experimental cup caused it to remove a greater depth of foam. Some specimens of lever-out removed only a half-ring, corresponding to the half-perimeter of the cup elevated during lever-out. Some surface damage on the inside of the GFRE cavities was observed with pull-out and lever-out tests, although of a much milder nature than the damage to the PU foam cavities.

Oversizing was found to affect the fixation stability

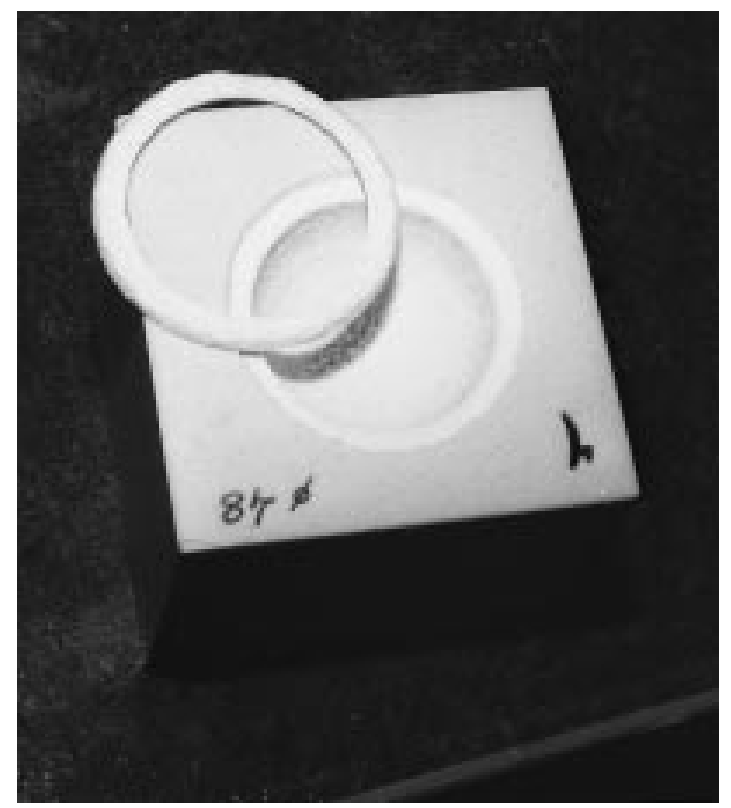

Fig. 7 Annulus of foam removed by cup rim at pull-out and lever-out
Table 2 Tukey's HSD test $p$ values for differences between cups

\begin{tabular}{lccl}
\hline & Optifix & PCA & H-G II \\
\hline Experimental & 0.0174 & 0.00015 & 0.00015 \\
Optifix & & 0.00015 & 0.00015 \\
PCA & & - & 0.032 \\
\hline
\end{tabular}

directly; reduced oversizing gave decreasing strengths in pull-out, lever-out and torque resistance in PU foams (Table 1). Multiple regression indicated that strengths were significantly correlated with oversizing for pullout $\left(R^{2}=0.61, p<0.02\right)$ and lever-out $\left(R^{2}=0.81\right.$, $p<0.0004)$. Including the effect of oversizing, PU foams still gave higher stability values than either GFRE cavities or cadaveric bone did.

Analysis of variance showed statistically significant differences for all main effects: cup design, test mode and cavity material. Differences identified by Tukey's HSD test were weaker between materials $(p=0.0013)$ than the differences due to test mode and between some of the cups [which were mostly at the $p<0.001$ level (Table 2)]. Weaker differences were found between the Optifix and the experimental cups $(p=0.017)$ and between the PCA and the $\mathrm{H}-\mathrm{G}$ II cups $(p=0.032)$. Multiple-regression testing for correlations with cups or test modes did not achieve statistical significance.

\section{DISCUSSION}

\subsection{Fixation modes}

The mode of fixation stability thought to be important depends on the mechanism assumed to cause acetabular failure. If 'particle disease' arising because wear particles infiltrate the fixation interface is the predominant mode, then the 'ability to engage' at the acetabular rim [4] will block ingress of particles by sealing off the mouth of the cavity; thus elastic grip at the acetabular rim is of paramount importance.

If the development of a fibrous interface follows micromotion at the interface, then the 'microstability' of the interface (its resistance to any motion during function) is primary, and the frictional properties of the interface are the key. These depend, in turn, on the coefficient of friction between the component surfaces and the bone surfaces, any contribution from the rim or edge, and the extent of elastic grip due to bone distortion about the cup surfaces. Grip at the rim or acetabular mouth features in both of these modes.

With increasing reliance on press-fit, some cups have been made less than a hemisphere to increase the bite of the rim. Fully hemispherical cups rely on 'elastic grip' in the acetabulum, i.e. isotropic expansion of the implant cavity inducing compressions of the bone against the cup surfaces. Since the acetabulum is not homogeneous or iso- 
tropic, the different elasticities of analogue test beds may enhance or confuse differences between cups due to design or surface finish. PU foams and GFRE cavities showed minor differences in trends between cups and tests.

The elastic deformation of the surrounding bone in turn relies on the properties and morphology of the bone and the difference between the final shape of the cavity (with the cup in situ) and its reamed shape and size. For this reason a separate study was undertaken to identify the variation in cavity size and shape introduced by the reaming technique [9]. Apart from accidental variation due to reaming error, deliberate misfitting by 'oversizing' affects the fixation stability of cups.

Cadaver bone shows greater variation in the measured parameters than both bone analogues for two reasons. Firstly, there is greater variation in the properties of bone between each individual case, meaning that the stiffness of the bone supporting the cup varies from case to case. Secondly, there is also variation in the fit of the cavity due to reaming error with the clinical technique [9], which alters the effective 'oversizing'.

\subsection{Oversizing}

In the present study, variation in the oversizing between $1.0,1.5$ and $2.0 \mathrm{~mm}$ showed a proportional increase in fixation strength in PU foams. This is in accordance with the work of Curtis et al. [2], who found $3 \mathrm{~mm}$ more stable, then $2 \mathrm{~mm}$ and then $1 \mathrm{~mm}$ of oversizing, and MacKenzie et al. [8], who found $2 \mathrm{~mm}$ oversizing to be the most stable. Although it might appear that increasing oversizing above $2 \mathrm{~mm}$ would improve stability, MacKenzie et al. found the gaps at the pole or 'equator' of the cup were greater with $4 \mathrm{~mm}$ oversizing than with $2 \mathrm{~mm}$; the strength of fit is increased but contact area is decreased.

Despite the difference in oversizing of the GFRE cavities used in the present study, there was a consistent trend between the GFRE results and other materials. If it had been possible to use $2 \mathrm{~mm}$ oversizing for the GFRE cavities, these trends might have been more clearly enhanced. Since stock-pultruded GFRE tubes were used here, the elastic deformation achievable with the cup impaction was limited. This could be overcome by using GFRE of a different modulus, or producing GFRE constructions of a more physiological form than the tubes used here.

\subsection{Test materials}

The torque and lever-out strength values measured in this series were similar to those reported in other studies in bone [4] and higher than in previously reported studies in PU foams [5]. Enhanced rim fixation increased stability in all modes.

Pull-out and lever-out tests of the PCA cup gave simi- lar results in PU foams to those measured in GFRE cavities. In contrast, the same measures in the Optifix and $\mathrm{H}-\mathrm{G}$ II cups were almost double in PU foams, and nearly tripled for the experimental cup. These three designs have much greater reliance on edge effect, which is particularly important in pull-out and lever-out testing. The half-rings of PU foam torn out with failure of these three cups in lever-out testing, and full rings with some specimens in pull-out tests, confirm the importance of the edge effect in these tests in this material. Some surface damage to the GFRE was observed during failure, suggesting a similar effect on a smaller scale.

The absolute strengths and differences between cups were lower in GFRE than in PU foams. This seems to be partly because cup friction against GFRE was different and partly because of the different mechanics of the model. The overall reduction in magnitude may also be due to the reduced oversizing in GFRE cavities $(1 \mathrm{~mm}$ instead of $2 \mathrm{~mm}$ ). Even with equal oversizing, the PU foams gave higher fixation strengths than either the GFRE cavities or cadaver bone did. With a different material and texture, the contribution of surface friction is greater than in bone or GFRE.

The stiffer harder GFRE also gives less frictional contribution to the fixation measures than the PU foam does, engaging less effectively with the surface roughness of the porous coating. Thus the comparative lever-out strengths show milder differences than in the PU foam, which probably better reflects the clinical performance. The lever-out strength of the experimental cup in cadaver bone was of the same order, suggesting that these results are closer to the physiological situation than are the PU foam measures.

The experimental cup showed greater stability in all properties than the other designs, except the torque resistance of the Optifix cup. This also depends on the frictional properties of the porous coating against the PU foam. Adler et al. [4] found that surface roughening features such as knurling, screw holes and shot peening (all of which increase the surface friction) enhanced the torsional stability. For this reason, an improved experimental cup was developed for clinical trials with increased frictional properties through surface roughening.

The differences between the effects of these designs are much less in GFRE cavities than in PU foams. It is considered that this is because the PU foam is softer and more compressible, increasing the friction against rough cup surfaces and allowing sharp or enhanced edges to dig in more. The GFRE is harder and engages the surface of the cups less; therefore the stability achieved is more affected by shape or form and is less sensitive to fine details such as edges. Because these cavities rely more on the stiffness of the supporting dense material (modelling the cortex) and shape effects, it is believed that this better models the elastic deformation occurring in the bone of the acetabulum due to impaction of an oversized cup into a prepared cavity. 
The acetabular analogue represented by a block of PU foam is that of a homogeneous isotropic mass of cancellous bone with a hemispherical cavity in one flat face. The true geometry and mechanics of the acetabulum are greatly different. In fact, the acetabulum can be represented better by a steep conical shell of cortical bone $[6,7,10-12]$, with some support from cancellous bone. On that understanding, a better model is a thin shell of material of similar stiffness to cortical bone, such as fibre-glass-reinforced plastics. Realistically, the interaction between the subchondral bone of the true acetabulum and the cortical shell of the pelvic face is complex, but a steep cone would better represent the contribution of the pelvic shell.

Clinically, acetabula are not reamed until all cortical bone is removed, but just until the subchondral bone has been sufficiently exposed that bleeding begins from its surfaces. This means that the subchondral shell still contributes to the mechanical stiffness of the acetabulum, reinforcing the outer shell which is the endosteum of the hemipelvis. Therefore the implanted acetabulum consists of the hemispherical subchondral shell supported by the conical endosteum, jointed at the rim of the acetabulum. Owing to the combination of these two cortical shells, an acceptable mechanical model of this stiffness might well be a cylindrical tube or hemispherical shell or cortical material as presented by the GFRE model used here.

In designs relying on 'press-fit' it is important to understand the relative contribution to initial stability of this elastic deformation, the surface friction and the edge effects. While PU foams may usefully highlight the role of some features in post-operative stability, owing to the great differences from the physiological mechanics they may lead to erroneous conclusions about relative cup performance.

\section{CONCLUSIONS}

1. GFRE cavities realistically model acetabular grip on uncemented cups, with a different mechanism from PU foam cavities.

2. Cup tests in several modes demonstrate differences in stability, as do different bone analogues, and usefully enhance our understanding of the principles of 'pressfit' fixation.

3. Cup shape and rim design contribute strongly to initial stability: the experimental cup with enhanced rim fixation demonstrated superior stability in all materials and most modes of testing.

\section{ACKNOWLEDGEMENTS}

This work was supported by the Swedish Association against Rheumatism, the Gothenburg Medical
Association, the Swedish Medical Association, the Askers Fond, the Hjalmar Svenssons Fond, the Neubergh Fond, and Astra Tech AB.

The central contribution of Stig Wennberg to the practical advancement of this work, his technical input and continued support in every way, are hereby acknowledged with gratitude. The advice and assistance of Anders Aspenberg in the development of the test methods were also significant. The assistance and support of the Histopathology and Orthopaedic Departments of Frenchay Hospital, Bristol, UK, are gratefully acknowledged, as are the continued help and encouragement of the members of the Department of Biomaterials/Handicap Research, Gothenburg.

\section{REFERENCES}

1 Clarke, H. J., Jinnah, R. H., Warden, K. E., Cox, Q. G. and Curtis, K. J. Evaluation of acetabular stability in uncemented prostheses. J. Arthroplasty, 1991, 6, 335-340.

2 Curtis, M. J., Hungerford, D. S., Jinnah, R. H. and Wilson, V. D. The initial stability of uncemented acetabular component. J. Bone Jt Surg., 1992, 74B, 372-376.

3 Lachiewicz, P. F., Suh, P. B. and Gilbert, J. A. In vitro initial fixation of porous-coated acetabular total hip components. J. Arthroplasty, 1989, 4, 201-205.

4 Adler, E., Stuchin, S. A. and Kummer, F. H. Stability of press-fit acetabular cups. J. Arthroplasty, 1992, 7, 295-301.

5 Kody, M. H., Dorey, F. J., Amstutz, H. C., Kabo, J. M. and Markolf, K. L. Strength of initial mechanical fixation of screw ring acetabular components. Clin. Orthop., 1990, 257, 146-153.

6 Jacob, H. A. C., Huggler, A. H., Dietschi, C. and Schreiber, A. Mechanical function of subchondral bone as experimentally determined on the acetabulum of the pelvis. J. Biomechanics, 1976, 9, 625-627.

7 Petersen, D. R., Crowninshield, R. D., Brand, R. A. and Johnston, R. C. An axisymmetric model of acetabular components in total hip arthroplasty. J. Biomechanics, 1982, 15, 305-315.

8 MacKenzie, J. R., Callaghan, J. J., Pedersen, D. R. and Brown, T. D. Areas of contact and extent of gaps with implantation of oversized acetabular components in total hip arthroplasty. Clin. Orthop., 1994, 298, 127-136.

9 Macdonald, W., Carlsson, L., Charnley, G. J., Jacobsson, M. and Johansson, C. B. Variability of acetabular reaming under surgical conditions. Transactions of the 43rd Annual Meeting of the Orthopaedic Research Society, Illinois, 1997, Vol. 22, p. 310.

10 Levenston, M. E., Beaupre, G. S., Schurman, D. J. and Carter, D. R. Computer simulations of stress-related bone remodeling around noncemented acetabular components. J. Arthroplasty, 1993, 8, 595-605.

11 O'Sullivan, G. S., Goodman, S. B. and Jones, H. H. Computerized tomographic evaluation of acetabular anatomy. Clin. Orthop., 1992, 277, 175-181.

12 Vasu, R., Carter, D. R. and Harris, W. H. Stress distributions in the acetabular region. 1: before and after total joint replacement. J. Biomechanics, 1982, 15, 155-164. 